Keywords: clear-cell renal cell carcinoma; interleukin-6; interleukin-6 receptor; prognostic biomarker; conditional survival; cancer-specific survival; organ-confined disease

\title{
Prognostic value of interleukin- 6 and interleukin-6 receptor in organ-confined clear-cell renal cell carcinoma: a 5-year conditional cancer-specific survival analysis
}

\author{
Qiang Fu ${ }^{1,5}$, Yuan Chang ${ }^{2,5}$, Huimin $\mathrm{An}^{3}$, Hangcheng $\mathrm{Fu}^{1}$, Yu Zhu ${ }^{3}$, Le Xu ${ }^{2}$, Weijuan Zhang ${ }^{\star, 4}$ and Jiejie $\mathrm{Xu}^{\star}, 1$ \\ ${ }^{1}$ Department of Biochemistry and Molecular Biology, School of Basic Medical Sciences, Fudan University, Shanghai, 200032, \\ China; ${ }^{2}$ Department of Urology, Zhongshan Hospital, Fudan University, Shanghai 200032, China; ${ }^{3}$ Department of Urology, \\ Shanghai Cancer Center, Shanghai Medical College, Fudan University, Mailbox 226, 138 Yixueyuan Road, Shanghai 200032, China \\ and ${ }^{4}$ Department of Immunology, School of Basic Medical Sciences, Fudan University, Shanghai 200032, China
}

Background: Interleukin-6 (IL-6) is the major cytokine that induces transcriptional acute and chronic inflammation responses, and was recently incorporated as a recurrence prognostication signature for localised clear-cell renal cell carcinoma (ccRCC). As the prognostic efficacy of initial risk factors may ebb during long-term practice, we aim to report conditional cancer-specific survival (CCSS) of RCC patients and evaluate the impact of IL-6 as well as its receptor (IL-6R) to offer more relevant prognostic information accounting for elapsing time.

Methods: We enrolled 180 histologically proven localised ccRCC patients who underwent nephrectomy between 2001 and 2004 with available pathologic information. Five-year CCSS was determined and stratified by future prognostic factors. Constant Cox regression analysis and Harrell's concordance index were used to indicate the predictive accuracy of established models.

Results: The 5-year CCSS of organ-confined ccRCC patients with both IL-6- and IL-6R-positive expression was 52\% at year 2 after surgery, which was close to locally advanced patients $(48 \%, P=0.564)$ and was significantly poorer than organ-confined patients with IL-6- or IL-6R-negative expression $(89 \%, P<0.001)$. Multivariate analyses proved IL-6 and IL-6R as independent predictors after adjusting for demographic factors. Concordance index of pT-IL-6-IL-6R risk stratification was markedly higher compared with the stage, size, grade and necrosis prognostic model $(0.724$ vs $0.669, P=0.002)$ or UCLA Integrated Staging System $(0.724$ vs 0.642 , $P=0.007$ ) in organ-confined ccRCC population during the first 5 years.

Conclusions: Combined IL-6 and IL-6R coexpression emerges as an independent early-stage immunologic prognostic factor for organ-confined ccRCC patients.

The incidence rates and mortality of renal cell carcinoma (RCC) in the United States have increased by more than $30 \%$ over the past two decades (Siegel et al, 2015). However, the outcomes of RCC differ greatly between diagnostic stage, tumour grade and histologic subtypes (Ljungberg et al, 2015). The 10-year CSS for localised clear-cell RCC (ccRCC; representing 65-75\% cases of
RCC) is only 55\% (Tilki et al, 2014). In the past 10 years, advanced tools have been developed to improve the clinical outcome prediction for localised RCC patients, such as the UCLA Integrated Staging System (UISS) and the stage, size, grade and necrosis (SSIGN) prognostic model (Frank et al, 2002; Zisman et al, 2002). However, the prognostic efficacy of those initial prognostic factors

\footnotetext{
${ }^{*}$ Correspondence: Dr W Zhang; E-mail: weijuanzhang@fudan.edu.cn or Dr J Xu; E-mail: jjxufdu@fudan.edu.cn
}

${ }^{5}$ These authors contributed equally to this work.

Received 22 June 2015; revised 11 August 2015; accepted 7 October 2015; published online 10 November 2015

(c) 2015 Cancer Research UK. All rights reserved 0007-0920/15 
may ebb during long-term practice because the emerging environmental risk factors and the following physical conditions after treatment may extensively affect the outcomes, and patients may be more interested in whether having outlived a period time when given an agent would benefit survival in the future. Therefore, the ability to adjust estimations on the basis of survival since treatment initiation or therapy duration would be clinically meaningful. Conditional survival, which is defined as the probability of surviving an additional time given a condition that the patients has already survived a specific time, can just provide this practical information over time.

Currently, several novel models have integrated molecular markers and aim to predict individual outcome, which differed with regard to the number and type of covariates, tool properties and end points (Leibovich et al, 2003; Brookman-May et al, 2013; Heng et al, 2013). As a well-known inflammatory cytokine, interleukin-6 (IL-6) has been implicated in a wide variety of human biological functions such as final steps of B-cells differentiation, haematopoiesis and platelet production, as well as acute and chronic inflammation-associated diseases including rheumatoid arthritis and cancers. Interleukin-6 was recently found to be secreted by RCC tumour cells and acts as an autocrine tumour growth factor to induce transcriptional inflammatory response and promote tumour progression through JAK-STAT pathway (Wolf et al, 2014). In fact, RCC is one of the typical diseases that act through extensive local infiltration of inflammatory cells. In a large prospective multicenter study, Negrier et al (2004) have reported that circulating IL-6 level is an important independent prognostic factor in metastatic RCC patients and could be helpful in personalised treatment. Our previous work also revealed that IL-11 receptor (which share high homology with IL-6R) predicted poor outcome of early-stage RCC ( $P a n$ et al, 2015). More recently, in the Lancet Oncology, Rini et al (2015) described the identification and validation of a 16-gene signature that showed improved recurrence prognostication in localised ccRCC patients when compared with Leibovich score. Among those 16 genes, only IL-6 was associated with the inflammation pathway; this highlighted the representative prognostic role of IL-6 in RCC (Rini et al, 2015).

The aim of our present study is to assess the intratumoral presence of IL-6/IL-6R expression in ccRCC patients and to determine their prognostic values through conditional survival analyses, which has been previously conducted in RCC, as well as to appreciate their potential clinical interests (Harshman et al, 2012).

\section{MATERIALS AND METHODS}

Patient population. The study database included 282 patients with RCC from Zhongshan Hospital, Fudan University, Shanghai, China. The primary inclusion criterion was pathologically proven RCC patients receiving surgery from 2001 to 2004. Thus, 465 eligible formalin-fixed paraffin-embedded (FFPE) specimens were obtained between 10 January 2001 and 30 December 2004, and 183 patients were excluded according to preset exclusion criteria (Supplementary Table S1). All valid patients received nephrectomy after diagnoses and none received radiotherapy or chemotherapy before surgery. To ensure consistent data collection, baseline demographic, clinical and laboratory data were collected simultaneously, MRI and CT scans were reassessed in radiology units and all archived diagnostic $\mathrm{H} \& \mathrm{E}$ slides were pathologically central reviewed by a pathologist (L Chen) independently. This study was approved by the institutional ethical review boards of the hospital and all patients stated informed consent along with phone call follow-up.

The primary outcome of interest was 5-year conditional cancerspecific survival (5-year CCSS). Cancer-specific survival (CSS) was defined as the time from surgery to death attributable to cancer according to clinicians and archived files; occurrences were censored if death was a result of something other than renal cancer or if the patient was alive at the end of follow-up. 5-Year conditional survival represents the probability that a patient with cancer will survive additional 5 years, given that the patient has already survived $x$ years (0-5 years in this report). For example, to compute the 5-year CCSS for patients who have survived $x$ years, calculate $(x+5)$-year cumulative CSS, which was limited to the patients who were still alive at the beginning of $x$ years (Zamboni et al, 2010).

Data collection. Follow-up data for all patients were obtained from the most recent medical review. All patients were examined routinely every 5-6 months during the first 5 years of follow-up and annually thereafter. The follow-up period ended in November 2014.

Survival and primary cancer data for all valid patients were included in our analyses. A standardised data form was created to retrieve all relevant information on sociodemographic data (age at surgery, gender), pathologic data (pathologic tumour, node, and metastasis status, tumour location), treatment-related data (type of resection or chemotherapy) and clinical data (presence of any malaises or symptoms). Histological subtypes were restratified according to 2014 EAU guidelines (Ljungberg et al, 2015). Tumour size was defined as the greatest tumour diameter based on pathological specimen. Tumour node metastasis staging was reclassified according to the 2010 AJCC TNM classification (Kim et al, 2011). Fuhrman grade, microvascular invasion (MVI), coagulative necrosis, sarcomatoid features and rhabdoid differentiation were reported according to the 2012 ISUP consensus (Delahunt et al, 2013). Symptomatic presentation and Eastern Cooperative Oncology Group performance status (ECOG-PS) were preoperatively recorded and rearchived as described previously (Karakiewicz et al, 2007). UCLA Integrated Staging System and SSIGN scores were applied to all valid patients according to the original scoring algorithm, respectively.

Statistical analysis. Associations between categorical and continuous clinicopathologic parameters vs IL-6/IL-6R expression groups were evaluated using the Fisher's exact test and Wilcoxon's rank-sum test, respectively. CSS was assessed and graphically illustrated using Kaplan-Meier or life-table method, and log-rank test was used for comparing different scoring categories. Independent associations between CSS and assessed clinicopathologic predictors were evaluated using multivariate Cox proportional hazards regression models, embodied by hazard ratios (HRs) and 95\% confidence intervals (95\% CIs) for each level of categories. The concordance index ( $\mathrm{C}$ index) was used to assess the predictive accuracy of different models, 0.5 is equivalent to toss of a coin and 1.0 represents ideal prediction (Harrell et al, 1996). The comparison between different C index was performed by Hanley-McNeil test (Hanley and McNeil, 1983). To reduce overfit bias and internally validate the predictive accuracy estimates, multivariable models and $\mathrm{C}$ index calculations were subjected to 1000 bootstrap resamples.

Statistical analyses were performed with SPSS, version 21.0 (IBM, Armonk, NY, USA), Stata SE, version 12.1 (Stata, College Station, TX, USA) and R software packages, version 3.1.2 (The R Foundation for Statistical Computing; http://www.r-project.org/). A two-sided $P$-value $<0.05$ was considered to be statistically significant for all reports.

Procedures. Primary FFPE RCC samples were obtained from the Department of Urology, Zhongshan Hospital of Fudan University with patients' consent and approval of the institutional review board of Fudan University. Microarray development and immunohistochemistry were performed according to the methods previously used (Xu et al, 2014), with appropriate antibodies after control staining (anti-IL-6 antibody, ab6672 (Abcam), diluted 1/100; anti-IL-6R antibody, ab128008 (Abcam), diluted 1/400). Immunohistochemistry sections and corresponding $\mathrm{H} \& \mathrm{E}$ sections were scanned by a fully 
Table 1. CSS estimates in relationship to prognostic variables and IL-6/IL-6R expression in localised ccRCC cohorts

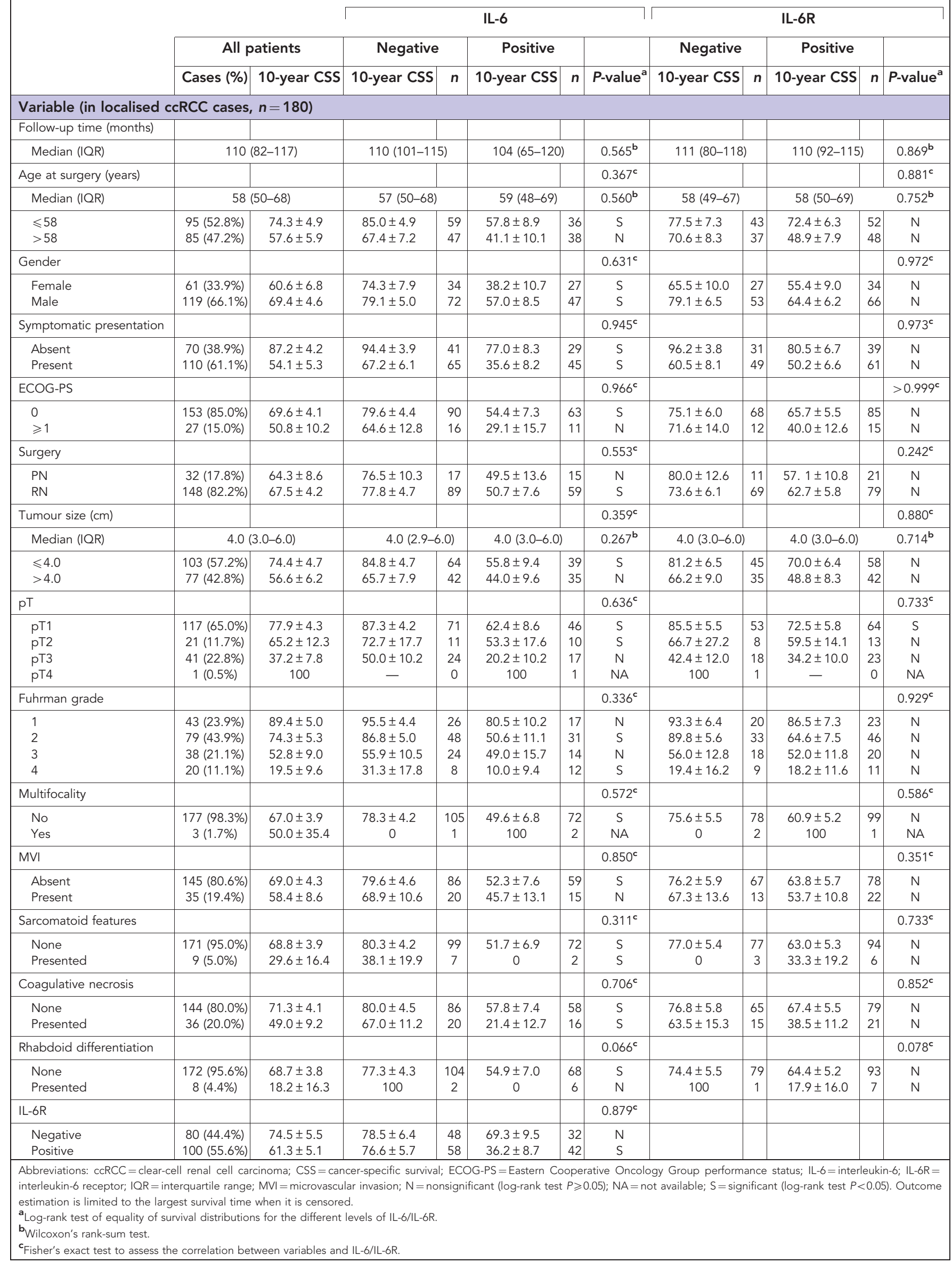


automated microscopy system (Leica DM6000 B; Leica Microsystems $\mathrm{GmbH}$, Mannheim, Germany), images were captured by Leica CVM2CL camera and analysed by Leica Ariol 4.0 software (Leica Microsystems $\mathrm{GmbH}$, Mannheim, Germany) automatically. For each sample, a total area of $4 \mathrm{~mm}^{2}$ was analysed (4 circulars from separate places of samples, each has $1 \mathrm{~mm}^{2}$ area). Cases were considered positive for expression when $>10 \%$ of tumour cells showed diffuse immunoreactivity.

\section{RESULTS}

Study population. The flow of patients through the study is shown in Supplementary Table S1. After applying initial exclusion criteria, 282 of 465 recruited patients were included in the analyses. No statistically significant differences in patient or tumour characteristics or survival outcomes were observed for patients with included $v s$ excluded data (Supplementary Table S2 and Supplementary Figure S1, online only).

The valid population included 198 males and 84 females, with a median age at surgery of 56 years (IQR, 49-67). Median follow-up of the survivors was 111 months (IQR 90-117, range 24-120, $n=282$ ). The morphological characteristics of patients are listed in Supplementary Table S5. One hundred and eighty-eight of 282 (66.7\%) patients were ccRCC accompanied with a poor 10-year CSS of $63.9 \%$; the number of these ccRCC specimens with positive IL-6 and IL-6R staining were 75 (39.9\%) and 105 (55.9\%), respectively.

Correlation analyses between prognostic variables, markers and outcome. We compared CSS between different IL-6/IL-6R

Table 2. 5-year CCSS and in relationship to IL-6 and IL-6R

5-year CCSS rates as time elapsed since surgery

\begin{tabular}{|l|c|c|c|c|c|c|}
\cline { 2 - 7 } & Baseline & 1 Year & 2 Year & 3 Year & 4 Year & 5 Year \\
\hline Cases, $n$ & 180 & 178 & 172 & 161 & 146 & 729 \\
\hline 5-year CCSS rates (\%) & $79.7 \pm 3.1$ & $75.3 \pm 3.4$ & $74.3 \pm 3.5$ & $75.0 \pm 3.6$ & $78.8 \pm 3.6$ & $83.8 \pm 3.6$ \\
\hline Survival gain (\%) & - & $-5.5 \pm 4.6$ & $-1.0 \pm 4.9$ & $+0.3 \pm 5.0$ & $+3.8 \pm 5.1$ & $+5.0 \pm 5.1$ \\
\hline Survival gain (\%) & - & $-5.5 \pm 4.6$ & $-5.4 \pm 4.7$ & $-4.7 \pm 4.7$ & $-0.9 \pm 4.7$ & $+4.1 \pm 4.7$
\end{tabular}

Age at surgery (years)

\begin{tabular}{|l|c|c|c|c|c|c|}
\hline$\leqslant 58$ & $81.7 \pm 4.0$ & $81.4 \pm 4.1$ & $83.7 \pm 4.0$ & $85.1 \pm 4.0$ & $86.6 \pm 4.0$ & $90.9 \pm 4.0$ \\
$>58$ & $77.3 \pm 4.7$ & $68.1 \pm 5.4$ & $63.5 \pm 5.7$ & $63.4 \pm 5.9$ & $68.8 \pm 6.2$ & $74.6 \pm 6.2$ \\
$P$-value & 0.545 & 0.094 & 0.007 & 0.004 & 0.019 & 0.007 \\
\hline
\end{tabular}

\begin{tabular}{|c|c|c|c|c|c|c|}
\hline \multicolumn{7}{|l|}{ Gender } \\
\hline Female & $75.9 \pm 5.6$ & $68.0 \pm 6.3$ & $68.2 \pm 6.4$ & $65.0 \pm 6.9$ & $71.8 \pm 7.0$ & $78.5 \pm 6.8$ \\
\hline Male & $81.6 \pm 3.6$ & $79.1 \pm 3.9$ & $77.3 \pm 4.1$ & $80.1 \pm 4.0$ & $82.2 \pm 4.1$ & $86.2 \pm 4.2$ \\
\hline$P$-value & 0.395 & 0.127 & 0.217 & 0.071 & 0.197 & 0.220 \\
\hline \multicolumn{7}{|l|}{ pT } \\
\hline $\mathrm{pT} 1+\mathrm{pT} 2$ & $86.2 \pm 3.0$ & $82.4 \pm 3.4$ & $82.7 \pm 3.4$ & $82.6 \pm 3.6$ & $84.9 \pm 3.5$ & $88.4 \pm 3.6$ \\
\hline $\mathrm{pT} 3+\mathrm{pT} 4$ & $59.4 \pm 7.6$ & $53.3 \pm 7.8$ & $48.0 \pm 8.1$ & $50.1 \pm 8.6$ & $56.2 \pm 9.6$ & $65.3 \pm 10.0$ \\
\hline$P$-value & $<0.001$ & $<0.001$ & $<0.001$ & $<0.001$ & 0.001 & 0.004 \\
\hline \multicolumn{7}{|l|}{ IL-6 } \\
\hline Negative & $88.2 \pm 3.2$ & $83.9 \pm 3.7$ & $80.7 \pm 4.0$ & $81.1 \pm 4.0$ & $85.0 \pm 3.9$ & $85.0 \pm 4.0$ \\
\hline Positive & $67.2 \pm 5.6$ & $62.6 \pm 6.0$ & $64.0 \pm 6.3$ & $63.8 \pm 6.9$ & $66.7 \pm 7.3$ & $66.3 \pm 8.0$ \\
\hline$P$-value & 0.001 & 0.001 & 0.012 & 0.020 & 0.011 & 0.027 \\
\hline \multicolumn{7}{|c|}{ Organ-confined subgroup (pT1 + pT2) } \\
\hline Negative & $94.8 \pm 2.5$ & $90.7 \pm 3.3$ & $89.3 \pm 3.6$ & $87.8 \pm 3.8$ & $89.6 \pm 3.7$ & $90.9 \pm 3.6$ \\
\hline Positive & $73.2 \pm 6.2$ & $69.5 \pm 6.7$ & $72.1 \pm 6.7$ & $72.7 \pm 7.3$ & $76.0 \pm 7.2$ & $83.3 \pm 7.8$ \\
\hline$P$-value & $<0.001$ & 0.001 & 0.008 & 0.034 & 0.030 & 0.424 \\
\hline \multicolumn{7}{|c|}{ Locally advanced subgroup (pT3 + pT4) } \\
\hline Negative & $66.7 \pm 9.6$ & $60.9 \pm 10.2$ & $52.2 \pm 10.4$ & $57.1 \pm 10.8$ & $66.7 \pm 11.1$ & $75.0 \pm 10.8$ \\
\hline Positive & $50.0 \pm 11.8$ & $43.8 \pm 11.9$ & $42.2 \pm 12.7$ & $40.2 \pm 13.6$ & $40.9 \pm 15.6$ & $50.0 \pm 17.7$ \\
\hline$P$-value & 0.259 & 0.206 & 0.495 & 0.372 & 0.280 & 0.376 \\
\hline \multicolumn{7}{|l|}{ IL-6R } \\
\hline Negative & $86.4 \pm 4.0$ & $80.4 \pm 4.7$ & $79.8 \pm 4.9$ & $80.0 \pm 5.0$ & $84.8 \pm 4.7$ & $87.9 \pm 3.6$ \\
\hline Positive & $74.6 \pm 4.4$ & $71.4 \pm 4.7$ & $69.9 \pm 4.9$ & $71.0 \pm 5.0$ & $73.9 \pm 5.2$ & $74.7 \pm 7.8$ \\
\hline$P$-value & 0.052 & 0.153 & 0.148 & 0.204 & 0.140 & 0.153 \\
\hline \multicolumn{7}{|c|}{ Organ-confined subgroup (pT1 + pT2) } \\
\hline Negative & $94.5 \pm 3.1$ & $90.5 \pm 4.1$ & $90.8 \pm 4.1$ & $89.5 \pm 4.5$ & $91.2 \pm 4.2$ & $90.9 \pm 3.6$ \\
\hline Positive & $80.0 \pm 4.6$ & $76.4 \pm 5.0$ & $76.5 \pm 5.1$ & $77.2 \pm 5.2$ & $79.8 \pm 5.3$ & $83.3 \pm 7.8$ \\
\hline$P$-value & 0.019 & 0.038 & 0.048 & 0.070 & 0.093 & 0.424 \\
\hline \multicolumn{7}{|c|}{ Locally advanced subgroup (pT3 + pT4) } \\
\hline Negative & $62.7 \pm 11.2$ & $51.3 \pm 11.7$ & $48.1 \pm 12.1$ & $51.0 \pm 12.5$ & $61.9 \pm 13.4$ & $75.0 \pm 10.8$ \\
\hline Positive & $56.5 \pm 10.3$ & $54.5 \pm 10.6$ & $47.6 \pm 10.9$ & $50.0 \pm 11.8$ & $52.5 \pm 13.1$ & $50.0 \pm 17.7$ \\
\hline$P$-value & 0.579 & 0.966 & 0.855 & 0.959 & 0.801 & 0.376 \\
\hline
\end{tabular}


expression subgroups following detailed histological subtypes. The results (Table 1) showed that IL-6 $(+)$ ccRCC patients had significantly poorer survival than IL-6 ( -$)$ ccRCC patients $(P=0.002)$, and IL-6R revealed the same patterns $(P=0.038)$. In view of the apparently different prognosis and treatment between localised and metastatic ccRCC, we analysed the relationships between IL-6/IL-6R and potential prognostic variables in localised patients (Table 1, lower panel) and metastatic patients (data not shown because of the limited cases, $n=8$ ). However, no correlation was noted here.

The impact of various prognostic factors on CCSS was assessed, and pathologic features including tumour size, pT, Fuhrman grade, symptomatic presentation, sarcomatoid features, rhabdoid differentiation and IL-6 expression were initially predictive for CCSS and progressively decreased over time (Supplementary Table S3). pT was the most significant predictor throughout this period. Consistent with the previous findings, IL-6R showed prognostic power only in organ-confined (OC) subgroups after stratification using $\mathrm{pT}$ (decreased over time and not significant after year 3), and IL-6 expression remained significant in OC subgroups (Table 2).

A

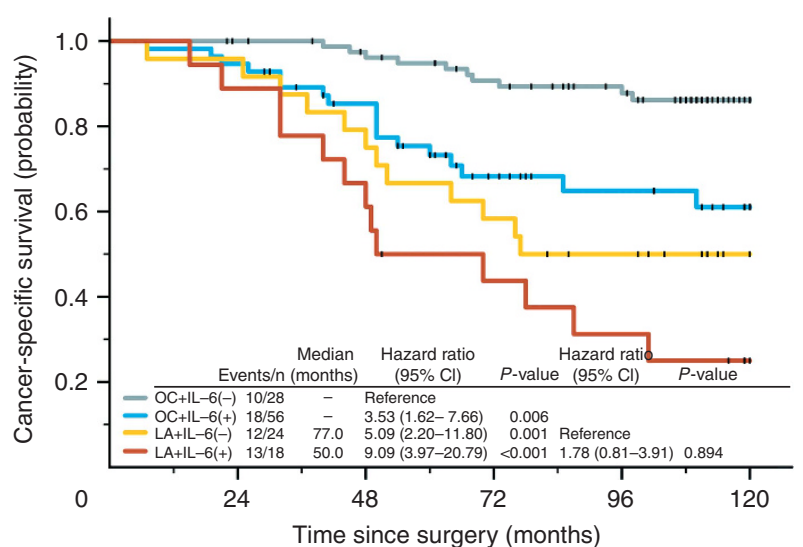

No. at risk

$\begin{array}{llllllllllll}\text { OC+IL-6(-) } & 82 & 82 & 80 & 79 & 74 & 72 & 66 & 62 & 56 & 36 & 0\end{array}$

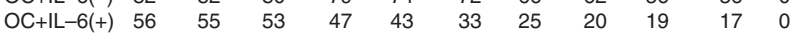
$\begin{array}{llllllllllll}\mathrm{LA}+\mathrm{IL}-6(-) & 24 & 23 & 23 & 21 & 18 & 16 & 14 & 11 & 10 & 6 & 0\end{array}$

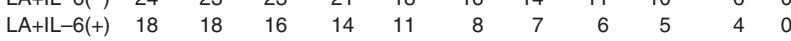

C

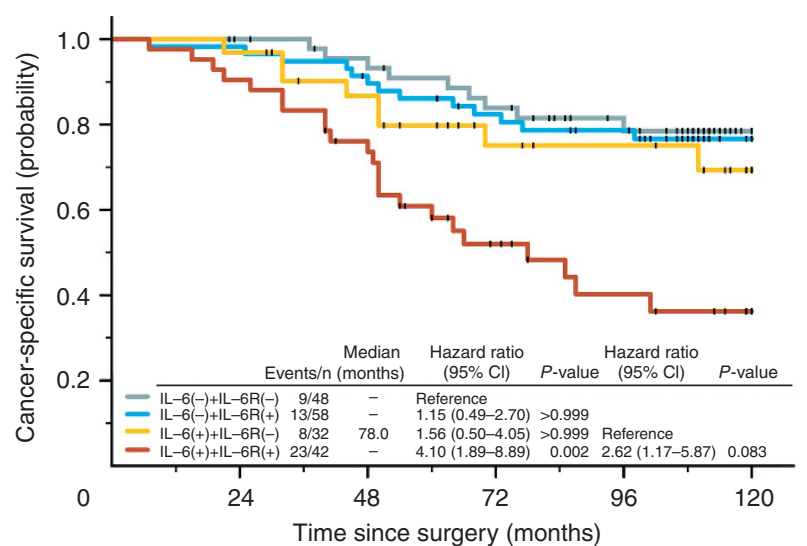

No. at risk

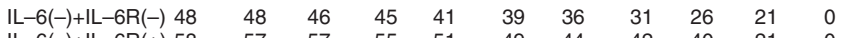

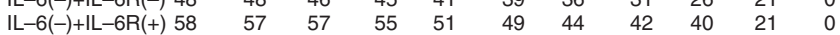

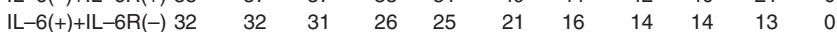

IL-6(+)+IL-6R(+) 42
To illustrate CSS estimates, 10-year Kaplan-Meier curves are shown in Figure 1. It shows that IL-6 $(-)$ significantly benefited patients' CSS in OC population $(P=0.006)$, but it was not the same case in locally advanced (LA) population $(P=0.894)$ (Figure $1 \mathrm{~A}$ and B). Furthermore, the strategy of combining IL-6 and IL-6R indicated that IL-6 $(+) / \mathrm{IL}-6 \mathrm{R}(+)$ patients experienced the shortest CSS (median $=78$ month) compared with any other groups (IL-6 (+)/IL-6R ( -$)$, IL-6 $(-) / \operatorname{IL}-6 \mathrm{R}(+)$, IL-6 ( - )/IL-6R $(-)$, median not reached) (Figure 1C). Interestingly, the OC patients with IL-6/IL-6R both positive shared the same CSS with LA patients $(P>0.999)$ (Figure 1D).

Multivariate analysis. The changing impact of independent parameters on CSS over time was assessed upon multivariable Cox regression analysis. In the crude full model, pT3-4 disease, high Fuhrman grade, MVI present, sarcomatoid features present, IL-6 $(+)$, and IL-6R $(+)$ were significantly associated with poorer survival at baseline; the effect of pathologic features on CSS decreased over time as illustrated by the decrease in HRs (Supplementary Table S4). We involved these factors in a reduced model adjusted by age and gender to control for their influence as

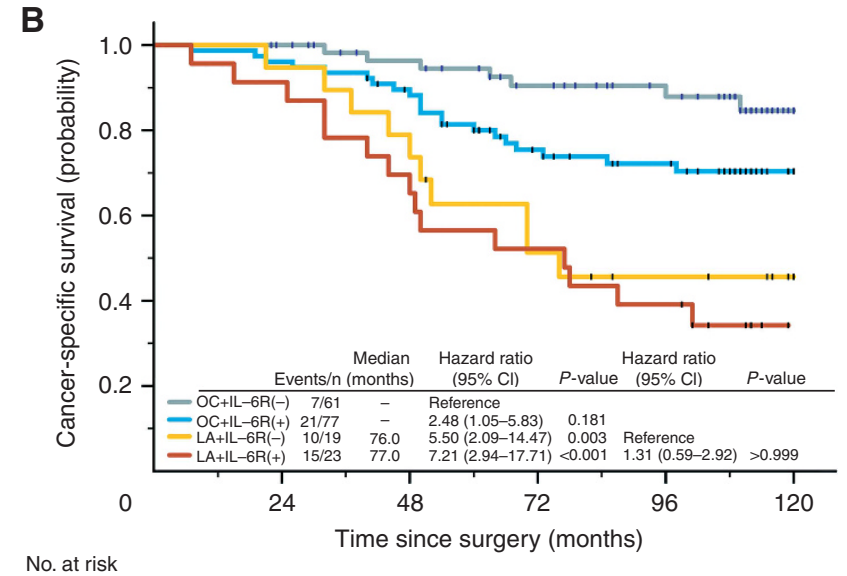

\begin{tabular}{|c|c|c|c|c|c|c|c|c|c|c|}
\hline $\mathrm{OC}+\mathrm{IL}-6 \mathrm{R}(-)$ & 61 & 61 & 59 & 54 & 52 & 49 & 43 & 38 & 34 & 29 \\
\hline $\mathrm{OC}+\mathrm{IL}-6 \mathrm{R}(+)$ & 77 & 76 & 74 & 72 & 65 & 56 & 48 & 44 & 41 & 24 \\
\hline $\mathrm{LA}+\mathrm{IL}-6 \mathrm{R}(-)$ & 19 & 19 & 18 & 17 & 14 & 11 & 9 & 7 & 6 & 5 \\
\hline LA+IL-6R(+) & 23 & 22 & 21 & 18 & 15 & 13 & 12 & 10 & 9 & 5 \\
\hline
\end{tabular}

D

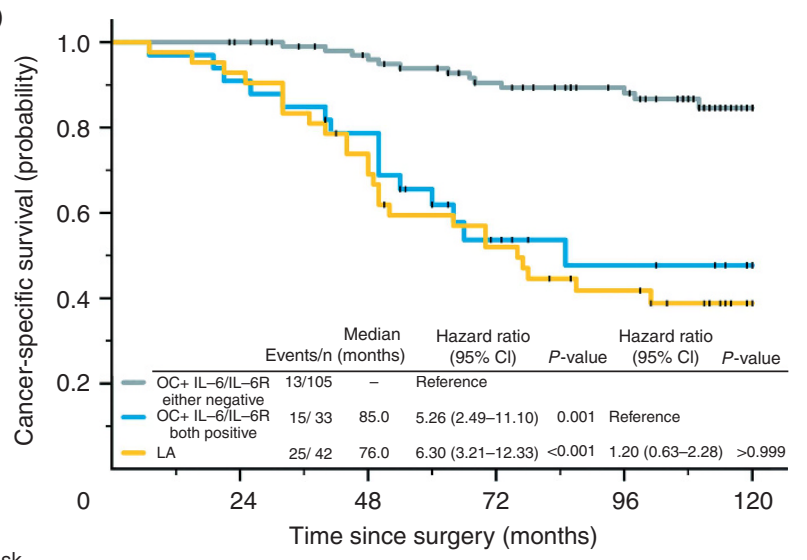

No. at risk

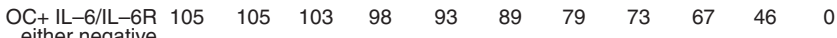

$\begin{array}{llllllllllll}\mathrm{OC}+\mathrm{IL}-6 / \mathrm{IL}-6 \mathrm{R} & 33 & 32 & 30 & 28 & 24 & 16 & 12 & 9 & 8 & 7 & 0\end{array}$

both positive

$\begin{array}{lllllllllll}42 & 41 & 39 & 35 & 29 & 24 & 21 & 17 & 15 & 10 & 0\end{array}$

Figure 1. Kaplan-Meier curves of 10-year cancer-specific survival for patients with localised ccRCC, stratified by (A) IL-6 and pT, (B) IL-6R and pT, (C) IL-6 and IL-6R and (D) IL-6, IL-6R and PT. To compare stratums in each panel, HR and 95\% Cl were obtained from univariate analyses for each categories, and relevant $P^{\prime}$-values were obtained from pairwise log-rank tests after Bonferroni correction. OA, organ-confined (pT1-2N0M0 stage disease); LA, locally advanced (pT3-4NOMO stage disease). 
covariates; IL-6 and IL-6R were still significant multivariate determinants of 5-year survival expectancy at surgery; the HR of IL-6R decreased from 2.69 to 2.02 and it fell out of the predictive factor list at year 3 (Table 3 ).

Comparison of SSIGN, UISS and the new recombination risk. Based on the above-mentioned findings, we considered IL6 , IL-6R and pT as synergetic predictors and incorporated them into a new recombination risk (termed ' $\mathrm{pT}$-IL-6-IL-6R risk'; low risk: OC patients with IL-6/IL-6R either negative; high risk: OC patients with IL-6/IL-6R both positive, and LA patients) (Table 4).
When stratified by UISS, SSIGN and pT-IL-6-IL-6R risk, the discriminations of 5-year CCSS were statistically significant at all time points $(P<0.001$ for all comparisons except that at year 5 of pT-IL-6-IL-6R risk), the relative risks for each model levels are shown in Figure 2A-D.

The predictive power for each models at any time point was assessed by C-index and further stratified by OC or LA population (Figure $2 \mathrm{E}$ and $\mathrm{F}$ and Supplementary Figure S2, online only). In the primary cohort, C-index of SSIGN or pT-IL-6-IL-6R risk were both higher compared with UISS and the gaps narrowed as time elapsed; no statistical difference was observed between pT-IL-6-IL-6R

Table 3. Reduced constant multivariable Cox regression analysis for predicting 5-year CCSS over time

\begin{tabular}{|c|c|c|c|c|c|c|}
\hline & \multicolumn{6}{|c|}{ Multivariable model as time elapsed since surgery } \\
\hline & Baseline & 1 Year & 2 Year & 3 Year & 4 Year & 5 Year \\
\hline \multicolumn{7}{|l|}{ Reduced Model ${ }^{a}$} \\
\hline $\begin{array}{l}\text { pT (pT3+pT4 vs pT1 + pT2) } \\
\text { HR } \\
95 \% \mathrm{Cl} \\
P \text {-value }\end{array}$ & $\begin{array}{c}4.35(+0.22) \\
2.10-9.02 \\
<0.001(0.001)\end{array}$ & $\begin{array}{c}4.03(+0.16) \\
2.06-7.88 \\
<0.001(0.001)\end{array}$ & $\begin{array}{c}4.40(+0.22) \\
2.27-8.52 \\
<0.001(0.001)\end{array}$ & $\begin{array}{c}4.29(+0.26) \\
2.13-8.66 \\
<0.001(0.001)\end{array}$ & $\begin{array}{c}4.76(+0.37) \\
2.07-10.94 \\
<0.001(0.002)\end{array}$ & $\begin{array}{c}3.86(+0.47) \\
1.42-10.45 \\
0.008(0.004)\end{array}$ \\
\hline $\begin{array}{l}\text { Fuhrman grade } \\
(3+4 \text { vs } 1+2) \\
\text { HR } \\
95 \% \mathrm{Cl} \\
\text { P-value }\end{array}$ & $\begin{array}{c}4.11(+0.46) \\
1.98-8.53 \\
<0.001(0.001)\end{array}$ & $\begin{array}{c}3.49(+0.27) \\
1.79-6.80 \\
<0.001(0.001)\end{array}$ & $\begin{array}{c}2.72(+0.18) \\
1.42-5.25 \\
0.003(0.001)\end{array}$ & $\begin{array}{l}2.53(+0.24) \\
1.26-5.07 \\
0.009(0.018)\end{array}$ & $\begin{array}{c}2.32(+0.22) \\
1.04-5.21 \\
0.041(0.041)\end{array}$ & $\begin{array}{c}2.08(+0.21) \\
0.73-5.95 \\
0.171(0.173)\end{array}$ \\
\hline $\begin{array}{l}\text { MVI (present vs absent) } \\
\text { HR } \\
95 \% \mathrm{Cl} \\
P \text {-value }\end{array}$ & $\begin{array}{l}2.20(+0.05) \\
1.05-4.62 \\
0.037(0.025)\end{array}$ & $\begin{array}{l}2.04(+0.07) \\
1.02-4.08 \\
0.044(0.047)\end{array}$ & $\begin{array}{c}1.83(+0.07) \\
0.90-3.72 \\
0.095(0.095)\end{array}$ & $\begin{array}{l}2.16(+0.12) \\
1.04-4.48 \\
0.038(0.051)\end{array}$ & $\begin{array}{c}2.34(+0.12) \\
0.99-5.52 \\
0.052(0.060)\end{array}$ & $\begin{array}{c}1.21(+0.01) \\
0.334-4.40 \\
0.770(0.734)\end{array}$ \\
\hline $\begin{array}{l}\text { Sarcomatoid features } \\
\text { (presented vs none) } \\
\mathrm{HR} \\
95 \% \mathrm{Cl} \\
P \text {-value }\end{array}$ & $\begin{array}{c}4.10(-0.75) \\
1.24-13.59 \\
0.021(0.010)\end{array}$ & $\begin{array}{c}4.32(-0.24) \\
1.44-12.91 \\
0.009(0.013)\end{array}$ & $\begin{array}{c}3.57(-0.57) \\
1.09-11.76 \\
0.036(0.050)\end{array}$ & $\begin{array}{c}4.06(-0.90) \\
1.18-14.00 \\
0.026(0.043)\end{array}$ & $\begin{array}{c}3.72(-2.71) \\
0.70-19.79 \\
0.123(0.101)\end{array}$ & $\begin{array}{c}4.36(-3.34) \\
0.74-25.82 \\
0.105(0.099)\end{array}$ \\
\hline $\begin{array}{l}\text { IL-6 (positive vs negative) } \\
\mathrm{HR} \\
95 \% \mathrm{Cl} \\
P \text {-value }\end{array}$ & $\begin{array}{c}4.17(+0.05) \\
1.97-8.83 \\
<0.001(0.001)\end{array}$ & $\begin{array}{c}3.58(+0.06) \\
1.81-7.07 \\
<0.001(0.001)\end{array}$ & $\begin{array}{c}2.59(+0.03) \\
1.35-4.99 \\
0.004(0.006)\end{array}$ & $\begin{array}{c}2.68(+0.01) \\
1.33-5.41 \\
0.006(0.009)\end{array}$ & $\begin{array}{l}2.95(+0.22) \\
1.32-6.59 \\
0.008(0.006)\end{array}$ & $\begin{array}{c}2.20(+0.04) \\
0.80-6.01 \\
0.125(0.105)\end{array}$ \\
\hline $\begin{array}{l}\text { IL-6R (positive vs negative) } \\
\text { HR } \\
95 \% \mathrm{Cl} \\
P \text {-value }\end{array}$ & $\begin{array}{l}2.69(+0.13) \\
1.27-5.69 \\
0.010(0.007)\end{array}$ & $\begin{array}{l}2.15(+0.06) \\
1.11-4.18 \\
0.024(0.025)\end{array}$ & $\begin{array}{l}2.05(+0.13) \\
1.06-3.99 \\
0.033(0.039)\end{array}$ & $\begin{array}{l}2.02(+0.17) \\
1.01-4.07 \\
0.047(0.066)\end{array}$ & $\begin{array}{l}2.27(+0.21) \\
0.98-5.27 \\
0.057(0.044)\end{array}$ & $\begin{array}{c}1.71(+0.29) \\
0.63-4.66 \\
0.294(0.289)\end{array}$ \\
\hline $\begin{array}{l}\text { Abbreviations: CCSS = conditional ca } \\
\text { multivariable models were calculatec } \\
\text { significant in the full model to be co } \\
a_{\text {Bootstrapped bias of HR is enclose }} \\
\text { Bootstrapped Cls are not shown her }\end{array}$ & $\begin{array}{l}\text { for covariate influe } \\
\text { in parentheses afte }\end{array}$ & $\begin{array}{l}\text { Bootstrapping with } \\
\text { HR value, and boo }\end{array}$ & $\begin{array}{l}\text { rd ratio; } \mathrm{IL}=\text { interlec } \\
\text { and gender by ea } \\
\text { resamples of the } \mathrm{s} \\
\text { apped } P \text {-value for }\end{array}$ & $\begin{array}{l}\text { ze of each time } p \\
\text { ariable at momer }\end{array}$ & $\begin{array}{l}\text { or; } \mathrm{MVI}=\text { microvas } \\
\text { re restricted in the } \\
\text { as used. } \\
\text { closed within pare }\end{array}$ & $\begin{array}{l}\text { invasion. The reduce } \\
\text { ables that considerec } \\
\text { ses after raw } P \text {-value }\end{array}$ \\
\hline
\end{tabular}

Table 4. pT-IL-6-IL-6R risk groups and CSS

\begin{tabular}{|c|c|c|c|c|c|c|c|}
\hline \multicolumn{8}{|c|}{ Prognostic group } \\
\hline Patient group & $\mathrm{pN} / \mathrm{M}$ & pT & IL-6 & IL-6R & No. (\%) & 5-year CSS (\%) & 10-year CSS (\%) \\
\hline pT-IL-6-IL-6R low risk & $\mathrm{pNO}$ and $\mathrm{MO}$ & $\begin{array}{l}1-2 \\
1-2 \\
1-2\end{array}$ & $\begin{array}{l}- \\
- \\
+\end{array}$ & $\begin{array}{l}- \\
+ \\
-\end{array}$ & $\begin{array}{l}38(20.2 \%) \\
44(23.4 \%) \\
23(12.2 \%)\end{array}$ & $\begin{array}{l}97.1 \pm 2.9 \\
93.1 \pm 3.9 \\
90.2 \pm 6.6\end{array}$ & $\begin{array}{l}87.2 \pm 6.0 \\
85.3 \pm 5.6 \\
81.2 \pm 10.4\end{array}$ \\
\hline pT-IL-6-IL-6R high risk & $\mathrm{pNO}$ and $\mathrm{MO}$ & $\begin{array}{l}1-2 \\
3-4 \\
3-4 \\
3-4 \\
3-4\end{array}$ & $\begin{array}{l}+ \\
- \\
- \\
+ \\
+\end{array}$ & $\begin{array}{l}+ \\
- \\
+ \\
- \\
+\end{array}$ & $\begin{array}{l}33(17.6 \%) \\
10(5.3 \%) \\
14(7.4 \%) \\
9(4.8 \%) \\
9(4.8 \%)\end{array}$ & $\begin{array}{l}61.9 \pm 8.7 \\
70.0 \pm 14.5 \\
64.3 \pm 12.8 \\
55.6 \pm 16.6 \\
44.4 \pm 16.6\end{array}$ & $\begin{array}{l}47.7 \pm 10.0 \\
50.0 \pm 15.8 \\
50.0 \pm 13.4 \\
41.7 \pm 17.3 \\
11.1 \pm 10.5\end{array}$ \\
\hline Metastatic & $\mathrm{pN} 1$ or $\mathrm{M} 1$ & Any & Any & Any & $8(4.3 \%)$ & $12.5 \pm 11.7$ & $12.5 \pm 11.7^{a}$ \\
\hline
\end{tabular}


A

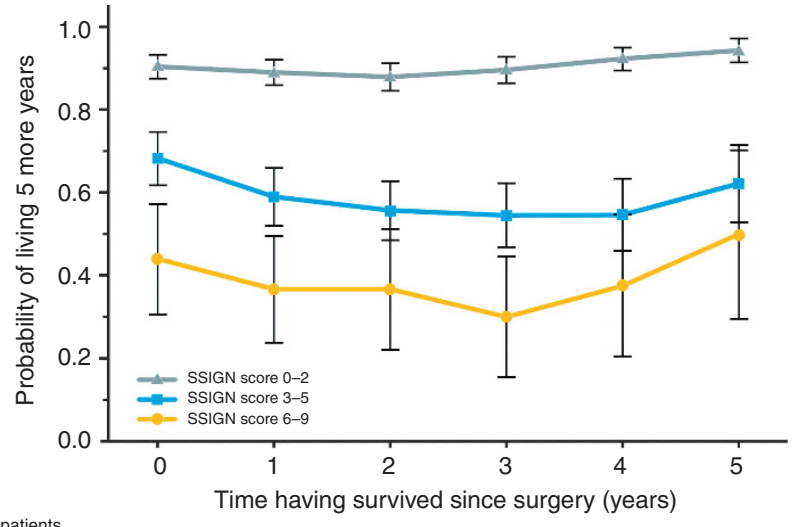

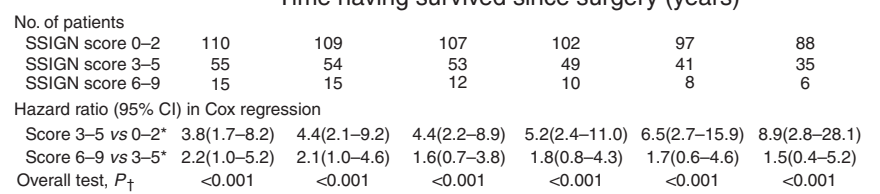

C
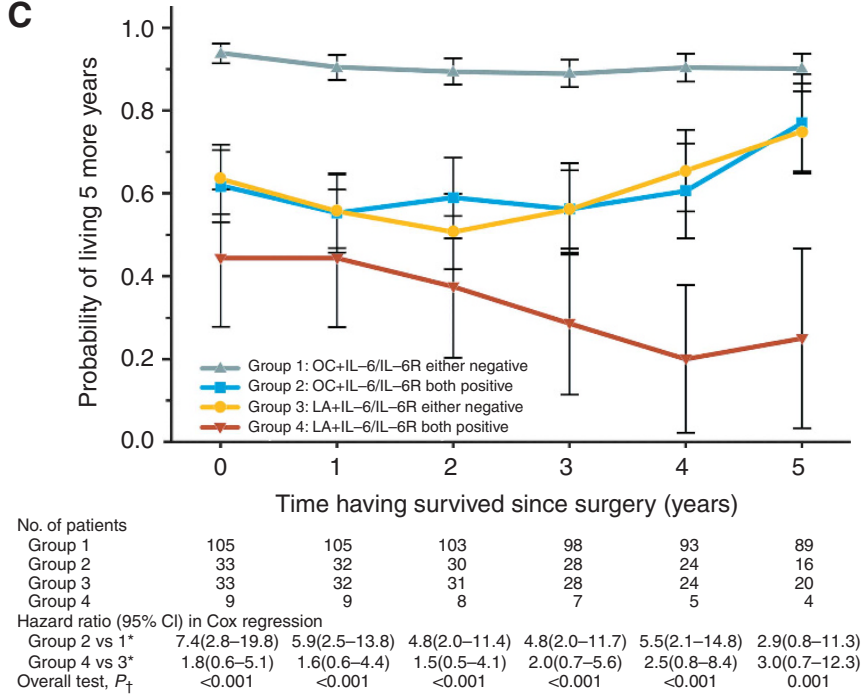

$\begin{array}{ccccccc}\text { Gerall test, } P_{\dagger} & 1.8(0.6-5.1) & 1.6(0.6-4.4) & 1.5(0.5-4.1) & 2.0(0.7-5.6) & 2.5(0.8-8.4) & 3.0(0.7-12.3) \\ \text { Ond } & <0.001 & <0.001 & <0.001 & <0.001 & 0.001\end{array}$

\section{$\mathbf{E}$}

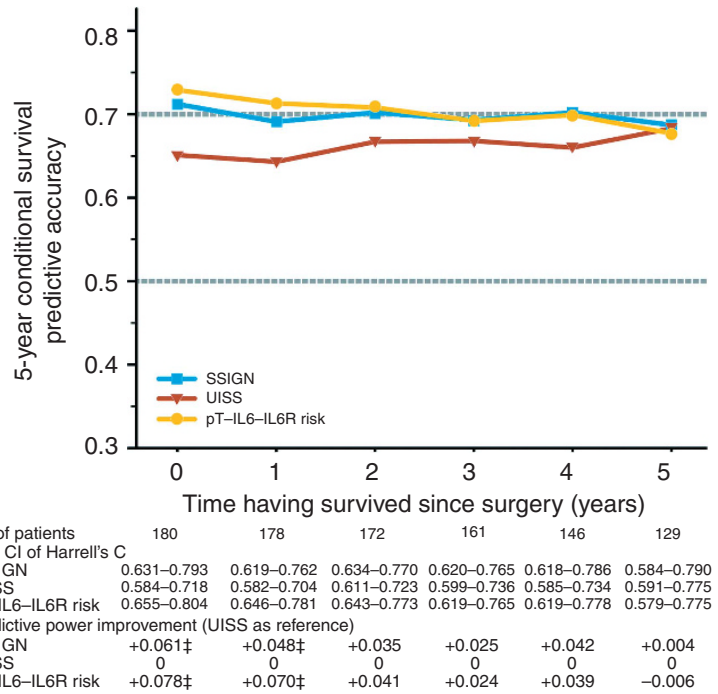

B

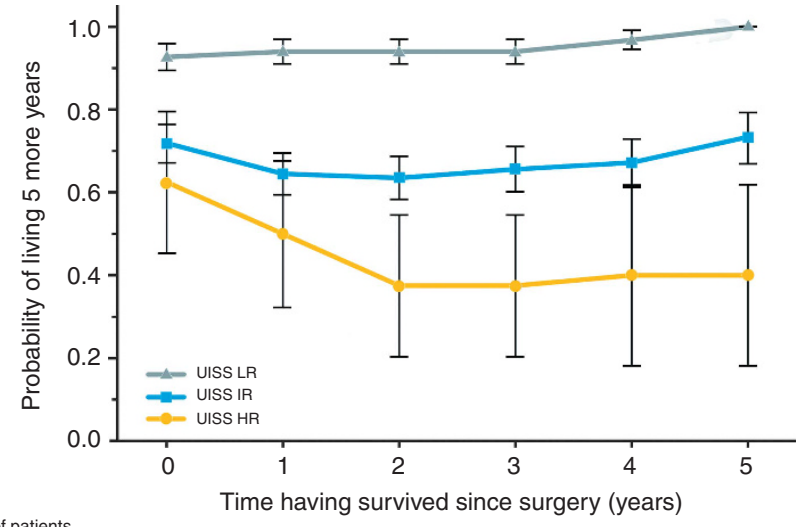

\begin{tabular}{lcccccc} 
No. of patients & \multicolumn{7}{c}{} \\
UISS LR & 73 & 72 & 70 & 68 & 64 & 58 \\
UISS IR & 99 & 98 & 94 & 85 & 77 & 66 \\
UISS HR & 8 & 8 & 8 & 8 & 5 & 5 \\
Hazard ratio & $(95 \%$ CI) in Cox regression & & & & \\
IR vs LR & $4.3(1.7-11.3)$ & $6.9(2.4-19.4)$ & $6.9(2.4-19.4)$ & $6.0(2.1-17.2)$ & $10.8(2.5-45.8)$ & - \\
HR vs IR* & $1.4(0.4-4.5)$ & $1.5(0.4-4.2)$ & $1.9(0.8-5.0)$ & $2.4(0.9-6.3)$ & $1.8(0.6-6.1)$ & $2.8(0.8-9.9)$ \\
Overall test, $P_{\dagger}$ & $<0.001$ & $<0.001$ & $<0.001$ & $<0.001$ & $<0.001$ & $<0.001$
\end{tabular}

D

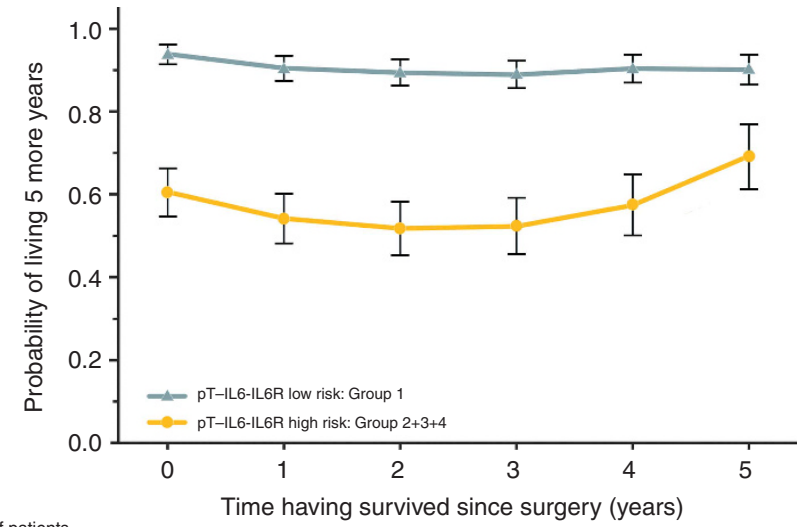

No. of patients

$\begin{array}{lrrrrrr}\text { PT-IL6-IL6R low risk } & 105 & 105 & 103 & 98 & 93 & 89 \\ \text { pT-IL6-IL6R high risk } & 75 & 73 & 69 & 63 & 53 & 40\end{array}$

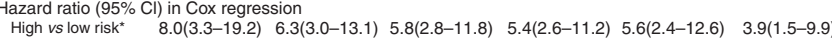

$\begin{array}{ccccccc}\text { High vs low risk } & 8.0(3.3-19.2) & 6.3(3.0-13.1) & 5.8(2.8-11.8) & 5.4(2.6-11.2) & 5.6(2.4-12.6) & 3.9(1.5-9.9) \\ \text { Overall test, } P_{\dagger} & <0.001 & <0.001 & <0.001 & <0.001 & <0.001 & 0.003\end{array}$

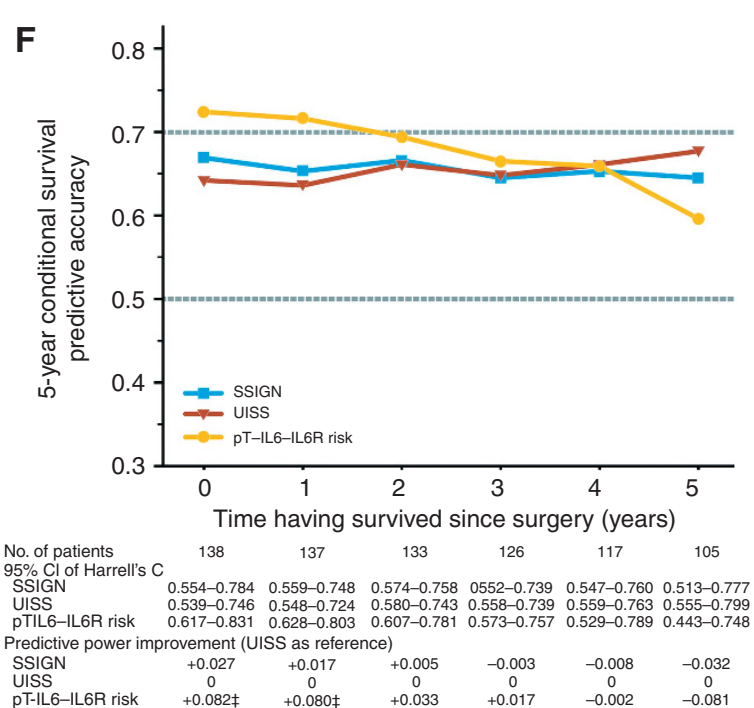

Figure 2. 5-year CCSS for all localised ccRCC patients stratified by (A) SSIGN, (B) UISS, (C) PT-IL-6-IL-6R risk, (D) pT-IL-6-IL-6R risk and the over-time discrimination of predictive power between these models (SSING, UISS and PT-IL-6-IL-6R risk) in (E) all population and (F) OC population. Harrell's concordance index (Harrell's C) and $95 \% \mathrm{Cl}$ obtained from 1000 bootstrap resamples was used to present the model's predictive accuracy. ${ }^{\star}$ The latter is the referential category. ${ }^{\dagger} P$-values were obtained from pooled log-rank tests over strata for 5 -year CCSS at each time point. ${ }^{\ddagger}$ Significant in Hanley-McNeil test $(P<0.05)$. HR, high risk; IR, intemediate risk; LR, low risk; UISS, the University of California Los Angeles Integrated Staging System; SSIGN, the stage, size, grade and necrosis. 
risk and SSIGN. In OC cohort, C-index of pT-IL-6-IL-6R risk was significantly higher compared with UISS or SSIGN at the beginning ( 0.724 vs 0.669 or 0.642 , respectively), and reached a low of 0.596 by year 5 (lower than SSIGN, C $=0.645$, and UISS, $\mathrm{C}=0.677$ ).

\section{DISCUSSION}

Interleukin-6 is a multifunctional cytokine with well-defined proand anti-inflammatory properties. It induces intracellular JAK/STAT or MAPK signalling pathways after binding to its receptor (IL-6R). Although the serum level of IL-6 in healthy humans is extremely low, it is highly and transiently upregulated in nearly all inflammationassociated pathophysiological states, including cancers. Alberti et al (2004) reported that IL-6 is produced at high levels by RCC cell lines and could activate the autocrine proliferation (Alberti et al, 2004). As IL-6/IL-6R signalling may be involved in the RCC progression, it was speculated that IL-6 and IL-6R could provide some prognostic information for clinical practice. Previous works have indicated the soluble IL-6 concentration before nephrectomy was a prognostic marker for CSS in localised RCC (Hrab et al, 2013). However, it should be more useful to assess the intratumoral expression of IL-6 for local tumour prognostication (e.g., detect IL-6 level in resected frozen tumour tissues), as IL-6 is secreted and signalled in an autocrine loop in RCC, and intratumoral IL-6 level should be significantly higher compared with that in plasma, where it preferentially represents the degree of systematic inflammation, but not local tumour progress.

In this study, we analysed the potential prognostic value of IL-6 and IL-6R in relation to 5-year CCSS, which is developed based on time-dependent covariate models. Conditional survival estimates may be more intriguing in clinical practice, especially in posttreatment prognostication, because the anatomical, histological and clinical characteristics acquired at diagnosis are generally a snapshot estimate of survival at the instant, and these factors may become less relevant with patient's survivorship as time goes on.

Our results show that, for patients with OC ccRCC who underwent nephrectomy, concurrence of IL-6- and IL-6R-positive expression in tumour tissue is an unfavorable prognostic factor in the early years. Organ-confined ccRCC patients who positively expressed both IL- 6 and IL-6R had 52\% probability of surviving an additional 5 years after living 2 years since surgery. This projection was similar with LA patients who had an average of $48 \%$ probability (Table 4). Interestingly, the discrimination of CCSS between pT-IL-6-IL-6R high- and low-risk subgroups appeared to fade as the time elapses from year 2, which may be caused by diminishing prognostic capability of IL-6R synchronously. Considering the unique in-series interaction between IL-6 and IL-6R (Figure 1C) and their self-evident affinity among transmembrane signalling, we audaciously speculated that the level of IL-6, rather than IL-6R, might decrease markedly since initial surgery. This is actually consistent with the fluctuating machinery of dynamic IL-6 behaviour in cancer-related chronic inflammation (Wolf et al, 2014).

Currently, nephrectomy remains the mainstay of localised RCC treatment, and some molecular biomarkers have been universally used in the management of RCC and had important roles in treatments. A precedent here is the application of IFN- $\alpha$ and IL-2 coupled with first-line targeted agents in novel immunotherapy for selected patients (Ljungberg et al, 2015). As some researches have revealed, IL- 6 has an important role in inflammatory cancer, and that these pathophysiological situations and events also affect the regulation of IL-6, IL-6 and IL-6R, which could serve as assistant prognostic markers for stratifying ccRCC patients with a high tumour burden to aid their personalised counselling and close follow-up, similar to the pT-IL-6-IL-6R risk model that we showed in the study, which could implement performance gains over some established prognostic models such as UISS and SSIGN in OC ccRCC. Meanwhile, IL-6/IL-6R serve as important upstream signal controllers of RCC proliferation or metastasis, as well as major communicators between tumours and immune microenvironments; they may be potential therapeutic targets for those high IL-6 level ccRCC patients once we develop a great understanding of their in vivo behaviours. As far as we known, the clinical phase II trials of siltuximab (an anti-IL-6 antibody) against tumourpromoting chronic inflammations are in progress, and tocilizumab (a humanised anti-IL-6R antibody) are also available in clinical practice (Nishimoto et al, 2009).

At least six different studies have shown that serum IL-6 levels are highly correlated with survival of RCC patients. However, two studies mainly focused on metastatic RCC (Blay et al, 1992; Ljungberg et al, 1997), another two had small sample size $(n<30)$ (Stadler et al, 1992; Yoshida et al, 2002) and one failed to assess the independent impact of IL-6 in multivariate analyses (Hrab et al, 2013). The final one revealed the correlation between outcome and in situ IL-6R presence with limited patients $(n=38)$ (Costes et al, 1997). All these detect IL-6 concentration in body fluids and none of them distinguish RCC pathologic variants meticulously. In fact, plasmic IL-6 is extremely sensitive but lack specificity to a particular disease (Wolf et al, 2014).

Our study is the first to assess intratumoral expression of IL-6 and IL-6R for localised ccRCC patients, and compare their prognostic power with established prognostic models of RCC. The application of conditional survival analysis also offers more deep-going investigations. However, our results were verified using an internal validation test (i.e., the bootstrap method), whereas a robust external cohort may be comparatively superior. Furthermore, it is difficult to identify the underlying mechanisms through which IL-6/IL-6R signalling regulate the carcinogenesis, progression and metastasis of ccRCC. An immunosuppressive effect on dendritic cells, a proinflammatory role through trans-signalling pathway, a growth factor effect and an epithelial-mesenchymal transition promoter role could all contribute to these observations; there are many works to accomplish before fully understanding the roles of IL-6 in RCC (Cabillic et al, 2006; Jones et al, 2011; Wolf et al, 2014; Zhang et al, 2015).

\section{ACKNOWLEDGEMENTS}

This study was funded by grants from National Basic Research Program of China (2012CB822104), National Natural Science Foundation of China $(31100629,31270863,81471621,81472227)$, Program for New Century Excellent Talents in University (NCET13-0146), and Shanghai Rising-Star Program (13QA1400300). All these study sponsors have no roles in the study design, in the collection, analysis and interpretation of data.

\section{CONFLICT OF INTEREST}

The authors declare no conflict of interest.

\section{REFERENCES}

Alberti L, Thomachot MC, Bachelot T, Menetrier-Caux C, Puisieux I, Blay JY (2004) IL-6 as an intracrine growth factor for renal carcinoma cell lines. Int J Cancer 111(5): 653-661.

Blay JY, Negrier S, Combaret V, Attali S, Goillot E, Merrouche Y, Mercatello A, Ravault A, Tourani JM, Moskovtchenko JF, Philip T, Favrot M (1992) Serum level of interleukin 6 as a prognosis factor in metastatic renal cell carcinoma. Cancer Res 52(12): 3317-3322. 
Brookman-May S, May M, Shariat SF, Xylinas E, Stief C, Zigeuner R, Chromecki T, Burger M, Wieland WF, Cindolo L, Schips L, De Cobelli O, Rocco B, De Nunzio C, Feciche B, Truss M, Gilfrich C, Pahernik S, Hohenfellner M, Zastrow S, Wirth MP, Novara G, Carini M, Minervini A, Simeone C, Antonelli A, Mirone V, Longo N, Simonato A, Carmignani G, Ficarra V. Members of the Cp, the Sp (2013) Features associated with recurrence beyond 5 years after nephrectomy and nephron-sparing surgery for renal cell carcinoma: development and internal validation of a risk model (PRELANE score) to predict late recurrence based on a large multicenter database (CORONA/SATURN Project). Eur Urol 64(3): 472-477.

Cabillic F, Bouet-Toussaint F, Toutirais O, Rioux-Leclercq N, Fergelot P, de la Pintiere CT, Genetet N, Patard JJ, Catros-Quemener V (2006) Interleukin- 6 and vascular endothelial growth factor release by renal cell carcinoma cells impedes lymphocyte-dendritic cell cross-talk. Clin Exp Immunol 146(3): 518-523.

Costes V, Liautard J, Picot MC, Robert M, Lequeux N, Brochier J, Baldet P, Rossi JF (1997) Expression of the interleukin 6 receptor in primary renal cell carcinoma. J Clin Pathol 50(10): 835-840.

Delahunt B, Cheville JC, Martignoni G, Humphrey PA, Magi-Galluzzi C, McKenney J, Egevad L, Algaba F, Moch H, Grignon DJ, Montironi R, Srigley JR. Members of the IRTP (2013) The International Society of Urological Pathology (ISUP) grading system for renal cell carcinoma and other prognostic parameters. Am J Surg Pathol 37(10): 1490-1504.

Frank I, Blute ML, Cheville JC, Lohse CM, Weaver AL, Zincke H (2002) An outcome prediction model for patients with clear cell renal cell carcinoma treated with radical nephrectomy based on tumor stage, size, grade and necrosis: the SSIGN score. J Urol 168(6): 2395-2400.

Hanley JA, McNeil BJ (1983) A method of comparing the areas under receiver operating characteristic curves derived from the same cases. Radiology 148(3): 839-843.

Harrell Jr FE, Lee KL, Mark DB (1996) Multivariable prognostic models: issues in developing models, evaluating assumptions and adequacy, and measuring and reducing errors. Stat Med 15(4): 361-387.

Harshman LC, Xie W, Bjarnason GA, Knox JJ, MacKenzie M, Wood L, Srinivas S, Vaishampayan UN, Tan MH, Rha SY, Donskov F, Agarwal N, Kollmannsberger C, North S, Rini BI, Heng DY, Choueiri TK (2012) Conditional survival of patients with metastatic renal-cell carcinoma treated with VEGF-targeted therapy: a population-based study. Lancet Oncol 13(9): 927-935.

Heng DY, Xie W, Regan MM, Harshman LC, Bjarnason GA, Vaishampayan UN, Mackenzie M, Wood L, Donskov F, Tan MH, Rha SY, Agarwal N, Kollmannsberger C, Rini BI, Choueiri TK (2013) External validation and comparison with other models of the International Metastatic Renal-Cell Carcinoma Database Consortium prognostic model: a population-based study. Lancet Oncol 14(2): 141-148.

Hrab M, Olek-Hrab K, Antczak A, Kwias Z, Milecki T (2013) Interleukin-6 (IL-6) and C-reactive protein (CRP) concentration prior to total nephrectomy are prognostic factors in localized renal cell carcinoma (RCC). Rep Pract Oncol Radiother 18(5): 304-309.

Jones SA, Scheller J, Rose-John S (2011) Therapeutic strategies for the clinical blockade of IL-6/gp130 signaling. J Clin Invest 121(9): 3375-3383.

Karakiewicz PI, Trinh QD, de la Taille A, Abbou CC, Salomon L, Tostain J, Cindolo L, Artibani W, Ficarra V, Patard JJ (2007) ECOG performance status 0 or 1 and symptom classification do not improve the ability to predict renal cell carcinoma-specific survival. Eur J Cancer 43(6): 1023-1029.

Kim SP, Alt AL, Weight CJ, Costello BA, Cheville JC, Lohse C, Allmer C, Leibovich BC (2011) Independent validation of the 2010 American Joint Committee on Cancer TNM classification for renal cell carcinoma: results from a large, single institution cohort. J Urol 185(6): 2035-2039.

Leibovich BC, Blute ML, Cheville JC, Lohse CM, Frank I, Kwon ED, Weaver AL, Parker AS, Zincke H (2003) Prediction of progression after radical nephrectomy for patients with clear cell renal cell carcinoma: a stratification tool for prospective clinical trials. Cancer 97(7): 1663-1671.

Ljungberg B, Bensalah K, Canfield S, Dabestani S, Hofmann F, Hora M, Kuczyk MA, Lam T, Marconi L, Merseburger AS, Mulders P, Powles T, Staehler M, Volpe A, Bex A (2015) EAU Guidelines on Renal Cell Carcinoma: 2014 Update. Eur Urol 67(5): 913-924.
Ljungberg B, Grankvist K, Rasmuson T (1997) Serum interleukin-6 in relation to acute-phase reactants and survival in patients with renal cell carcinoma. Eur J Cancer 33(11): 1794-1798.

Negrier S, Perol D, Menetrier-Caux C, Escudier B, Pallardy M, Ravaud A, Douillard JY, Chevreau C, Lasset C, Blay JY, Groupe Francais dI (2004) Interleukin-6, interleukin-10, and vascular endothelial growth factor in metastatic renal cell carcinoma: prognostic value of interleukin-6 - from the Groupe Francais d'Immunotherapie. J Clin Oncol 22(12): 2371-2378.

Nishimoto N, Miyasaka N, Yamamoto K, Kawai S, Takeuchi T, Azuma J (2009) Long-term safety and efficacy of tocilizumab, an anti-IL-6 receptor monoclonal antibody, in monotherapy, in patients with rheumatoid arthritis (the STREAM study): evidence of safety and efficacy in a 5-year extension study. Ann Rheum Dis 68(10): 1580-1584.

Pan D, Xu L, Liu H, Zhang W, Zhu Y, Xu J, Gu J (2015) Interleukin-11 receptor predicts post-operative clinical outcome in patients with early-stage clear-cell renal cell carcinoma. Jpn J Clin Oncol 45(2): 202-209.

Rini B, Goddard A, Knezevic D, Maddala T, Zhou M, Aydin H, Campbell S, Elson P, Koscielny S, Lopatin M, Svedman C, Martini JF, Williams JA, Verkarre V, Radulescu C, Neuzillet Y, Hemmerle I, Timsit MO, Tsiatis AC, Bonham M, Lebret T, Mejean A, Escudier B (2015) A 16-gene assay to predict recurrence after surgery in localised renal cell carcinoma: development and validation studies. Lancet Oncol 16(6): 676-685.

Siegel RL, Miller KD, Jemal A (2015) Cancer statistics, 2015. CA Cancer J Clin 65(1): 5-29.

Stadler WM, Richards JM, Vogelzang NJ (1992) Serum interleukin-6 levels in metastatic renal cell cancer: correlation with survival but not an independent prognostic indicator. J Natl Cancer Inst 84(23): 1835-1836.

Tilki D, Nguyen HG, Dall'Era MA, Bertini R, Carballido JA, Chromecki T, Ciancio G, Daneshmand S, Gontero P, Gonzalez J, Haferkamp A, Hohenfellner M, Huang WC, Koppie TM, Lorentz CA, Mandel P, Martinez-Salamanca JI, Master VA, Matloob R, McKiernan JM, Mlynarczyk CM, Montorsi F, Novara G, Pahernik S, Palou J, Pruthi RS, Ramaswamy K, Rodriguez Faba O, Russo P, Shariat SF, Spahn M, Terrone C, Vergho D, Wallen EM, Xylinas E, Zigeuner R, Libertino JA, Evans CP (2014) Impact of histologic subtype on cancer-specific survival in patients with renal cell carcinoma and tumor thrombus. Eur Urol 66(3): $577-583$.

Wolf J, Rose-John S, Garbers C (2014) Interleukin-6 and its receptors: a highly regulated and dynamic system. Cytokine 70(1): 11-20.

Xu L, Zhu Y, An H, Liu Y, Lin Z, Wang G, Xu J (2014) Clinical significance of tumor-derived IL-1beta and IL-18 in localized renal cell carcinoma: Associations with recurrence and survival. Urol Oncol 33(2): 68.e9-16.

Yoshida N, Ikemoto S, Narita K, Sugimura K, Wada S, Yasumoto R, Kishimoto T, Nakatani T (2002) Interleukin-6, tumour necrosis factor alpha and interleukin-1beta in patients with renal cell carcinoma. Br J Cancer 86(9): 1396-1400.

Zamboni BA, Yothers G, Choi M, Fuller CD, Dignam JJ, Raich PC, Thomas Jr CR, O'Connell MJ, Wolmark N, Wang SJ (2010) Conditional survival and the choice of conditioning set for patients with colon cancer: an analysis of NSABP trials C-03 through C-07. J Clin Oncol 28(15): 2544-2548.

Zhang M, Gong W, Zhang Y, Yang Y, Zhou D, Weng M, Qin Y, Jiang A, Ma F, Quan Z (2015) Expression of interleukin-6 is associated with epithelial-mesenchymal transition and survival rates in gallbladder cancer. Mol Med Rep 11(5): 3539-3546.

Zisman A, Pantuck AJ, Wieder J, Chao DH, Dorey F, Said JW, deKernion JB, Figlin RA, Belldegrun AS (2002) Risk group assessment and clinical outcome algorithm to predict the natural history of patients with surgically resected renal cell carcinoma. J Clin Oncol 20(23): 4559-4566.

This work is published under the standard license to publish agreement. After 12 months the work will become freely available and the license terms will switch to a Creative Commons AttributionNonCommercial-Share Alike 4.0 Unported License. 